\title{
Another exact solution for two-dimensional, inviscid sinh Poisson vortex arrays
}

\author{
K. W. Chow, ${ }^{\text {a) }}$ S. C. Tsang, and C. C. Mak \\ Department of Mechanical Engineering, University of Hong Kong, Pokfulam Road, Hong Kong
}

(Received 8 November 2002; accepted 25 April 2003; published 2 July 2003)

\begin{abstract}
Arrays of vortices are considered for two-dimensional, inviscid flows when the functional relationship between the stream function and the vorticity is a hyperbolic sine. An exact solution as a doubly periodic array of vortices is expressed in terms of the Jacobi elliptic functions. There is a threshold value of the period parameter such that a transition from globally smooth distributions of vorticity to singular distributions occurs. (c) 2003 American Institute of Physics.
\end{abstract}

[DOI: $10.1063 / 1.1584046]$

One possible mode of two-dimensional, incompressible, inviscid, steady flows free of body forces is governed by the relation

$$
\nabla^{2} \psi=\psi_{x x}+\psi_{y y}=f(\psi)=-\omega .
$$

$\psi$ is the stream function and $\omega$ is the vorticity. $f$ is a differentiable but otherwise arbitrary function. The choice

$$
f(\psi)=-\sigma \sinh \psi=\nabla^{2} \psi, \quad(\sigma>0)
$$

is known as the sinh Poisson equation ${ }^{1-7}(\operatorname{shP})$, and serves as a model in the studies of the most probable state in inviscid two-dimensional flows in fluids and plasmas. Exact solutions are thus of fundamental fluid dynamical interests as well as of relevance in applications. Examples from the literature include:

(a) the Mallier-Maslowe vortices ${ }^{2}$

$$
\psi=4 \tanh ^{-1}\left[\frac{\beta \cos \left(\sqrt{1+\beta^{2}} x\right)}{\sqrt{1+\beta^{2}} \cosh (\beta y)}\right], \quad \sigma=1 ;
$$

(b) a doubly periodic array of vortices in terms of products of elliptic functions ${ }^{8}$

$$
\begin{aligned}
& \psi=4 \tanh ^{-1}\left[\left(\frac{s k_{1}}{r \sqrt{1-k^{2}}}\right) \operatorname{cn}(r x, k) \operatorname{cn}\left(s y, k_{1}\right)\right], \\
& r^{4} k^{2}\left(1-k^{2}\right)=s^{4} k_{1}^{2}\left(1-k_{1}^{2}\right), \\
& r^{2}\left(1-2 k^{2}\right)+s^{2}\left(1-2 k_{1}^{2}\right)=\sigma ;
\end{aligned}
$$

(c) doubly periodic arrays of vortices in terms of rational expressions of elliptic functions ${ }^{6,9}$

$$
\begin{aligned}
& \text { (i) } \quad \psi=4 \tanh ^{-1}\left[\frac{\sqrt{k} \operatorname{sn}(r x, k)-\sqrt{k_{1}} \operatorname{sn}\left(s y, k_{1}\right)}{1+\sqrt{k k_{1}} \operatorname{sn}(r x, k) \operatorname{sn}\left(s y, k_{1}\right)}\right], \\
& s^{2}\left(1-k_{1}\right)^{2}=1+4 r^{2} k, \quad s\left(1+k_{1}\right)=r(1+k), \\
& \sigma=1,
\end{aligned}
$$

(ii)

$$
\begin{aligned}
& \psi=4 \tanh ^{-1}\left[\frac{\operatorname{dn}(r x, k) \operatorname{dn}\left(s y, k_{1}\right)-\left(1-k^{2}\right)^{1 / 4}\left(1-k_{1}^{2}\right)^{1 / 4}}{\left(1-k_{1}^{2}\right)^{1 / 4} \operatorname{dn}(r x, k)+\left(1-k^{2}\right)^{1 / 4} \operatorname{dn}\left(s y, k_{1}\right)}\right] \\
& r\left(1+\sqrt{1-k^{2}}\right)=s\left(1+\sqrt{1-k_{1}^{2}}\right), \quad \sigma=-r^{2}\left(1-\sqrt{1-k^{2}}\right)^{2}+4 s^{2} \sqrt{1-k_{1}^{2}}
\end{aligned}
$$

The main goal of the present work is to present another exact solution for shP. Equation (2) is first rewritten in bilinear form

$$
\begin{aligned}
& \psi=4 \tanh ^{-1}\left(\frac{g}{f}\right), \\
& \left(D_{x}^{2}+D_{y}^{2}-C_{0}\right)(g \cdot g+f \cdot f)=0, \\
& \left(D_{x}^{2}+D_{y}^{2}-C_{0}+\sigma\right) g \cdot f=0 .
\end{aligned}
$$

a)Telephone: (852) 2859 2641; fax: (852) 2858 5415. Electronic mail: kwchow@hkusua.hku.hk
$C_{0}$ is a constant and $D$ is the Hirota bilinear operator defined by

$$
\begin{aligned}
D_{x}^{m} D_{t}^{n} g \cdot f= & \left(\frac{\partial}{\partial x}-\frac{\partial}{\partial x^{\prime}}\right)^{m}\left(\frac{\partial}{\partial t}-\frac{\partial}{\partial t^{\prime}}\right)^{n} \\
& \times\left. g(x, t) f\left(x^{\prime}, t^{\prime}\right)\right|_{x=x^{\prime}, t=t^{\prime}} .
\end{aligned}
$$

The bilinear forms are known to be effective in obtaining solitary and periodic waves for nonlinear equations. ${ }^{6,10}$

A doubly periodic array of vortices. Periodic solutions can now be derived by searching for $g$ and $f$ in terms of products of elliptic and theta functions. ${ }^{11}$ The methodology is 


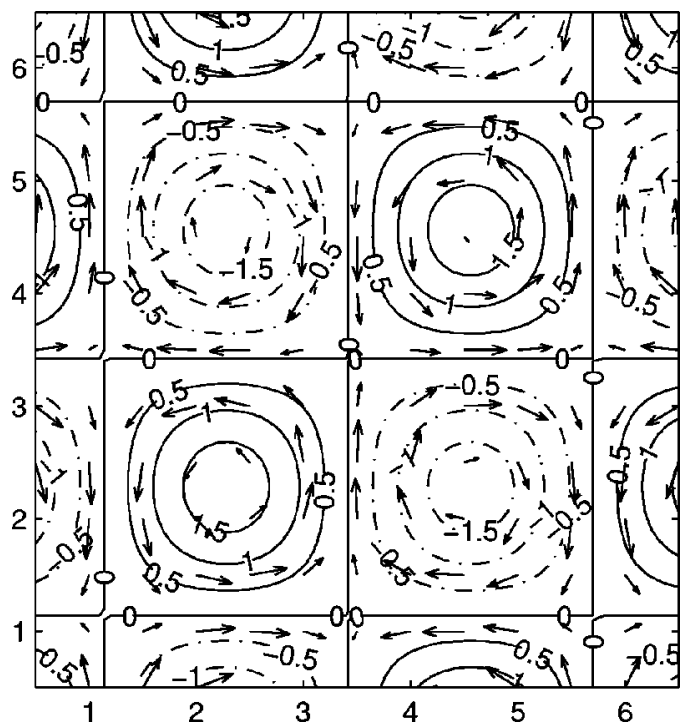

FIG. 1. Streamlines for the doubly periodic arrays of vortices, $r=s=1, k$ $=0.9$ (dotted lines denote negative values of the stream function).

very similar to the one used in our earlier work, ${ }^{6}$ and hence only the final results will be stated here. A product periodic solution for the sinh Poisson equation is

$$
\begin{aligned}
\psi= & 4 \tanh ^{-1}\left\{A_{0}\left[\frac{\operatorname{dn}(r x, k)}{\left(1-k^{2}\right)^{1 / 4}}-\frac{\left(1-k^{2}\right)^{1 / 4}}{\operatorname{dn}(r x, k)}\right]\right. \\
& \left.\times\left[\frac{\operatorname{dn}\left(s y, k_{1}\right)}{\left(1-k_{1}^{2}\right)^{1 / 4}}-\frac{\left(1-k_{1}^{2}\right)^{1 / 4}}{\operatorname{dn}\left(s y, k_{1}\right)}\right]\right\},
\end{aligned}
$$

where the amplitude parameter is defined by

$$
A_{0}=\frac{s\left(1-k_{1}^{2}\right)^{1 / 4}}{2 r\left(1-\sqrt{1-k^{2}}\right)} .
$$

The wavenumbers and the moduli of the elliptic functions, $k$, $k_{1}$, are related by

$$
s^{2}\left(1-k_{1}^{2}\right)^{1 / 4}\left[1-\sqrt{1-k_{1}^{2}}\right]=r^{2}\left(1-k^{2}\right)^{1 / 4}\left[1-\sqrt{1-k^{2}}\right] .
$$

The vorticity parameter, $\sigma$ [Eq. (2)], is given by

$$
\sigma=r^{2}\left[6 \sqrt{1-k^{2}}-2+k^{2}\right]+s^{2}\left[6 \sqrt{1-k_{1}^{2}}-2+k_{1}^{2}\right] \text {. }
$$

We verify by direct differentiation that Eqs. (8)-(11) satisfy Eq. (2) using the computer algebra software MATHEMATICA.

Figure 1 shows that Eq. (8) in general represents again a doubly periodic array of vortices. The direction of rotation in each cell is different from that in the adjacent cells. The Jacobi elliptic function $\operatorname{dn}(x)$ has period $2 K$ where $K$ is the complete elliptic integral of the first kind

$$
K(k)=\int_{0}^{\pi / 2} \frac{d \theta}{\sqrt{1-k^{2} \sin ^{2} \theta}} .
$$

Moduli of the elliptic functions $k, k_{1}$ will be used below to represent the periods of the doubly periodic arrays of vortices.

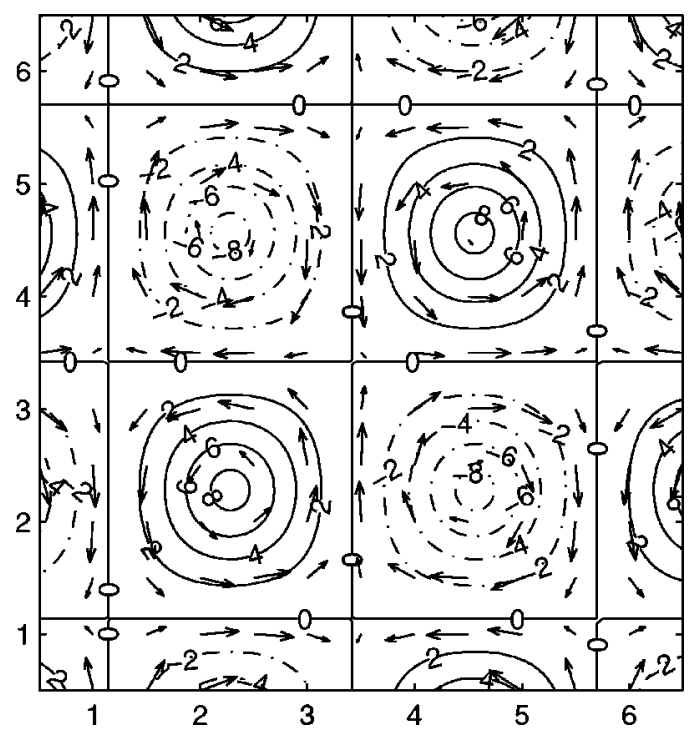

FIG. 2. Lines of constant vorticity for the doubly periodic arrays of vortices, $r=s=1, k=0.9$ (dotted lines denote negative values of the vorticity).

The boundaries of the cells are horizontal and vertical lines defined by the equations

$$
\operatorname{dn}(r x, k)=\left(1-k^{2}\right)^{1 / 4}, \quad \operatorname{dn}\left(s y, k_{1}\right)=\left(1-k_{1}^{2}\right)^{1 / 4} .
$$

The vorticity within each cell (Fig. 2) can be related to the circulation around the cell boundaries

$$
\oint u d x+v d y,
$$

by the Stokes theorem. Analytical expressions are feasible by identities of elliptic functions, but the details will not be pursued here.

A nearly circular singularity in vorticity. In general values of the stream function will get larger as $k$ or $k_{1}$ approaches one (Fig. 3). The intriguing aspect of the present

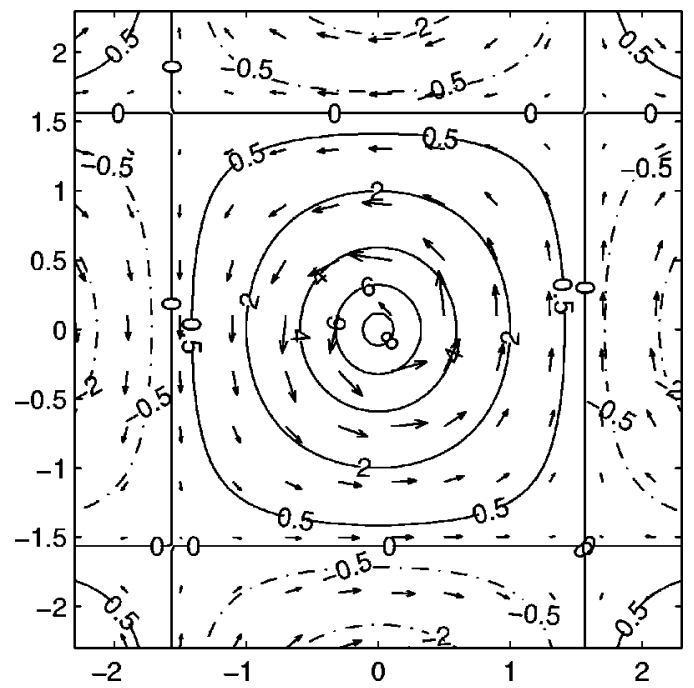

FIG. 3. Streamlines in a cell near the threshold value of $k, r=s=1, k$ $=0.984$ (dotted lines denote negative values of the stream function). 


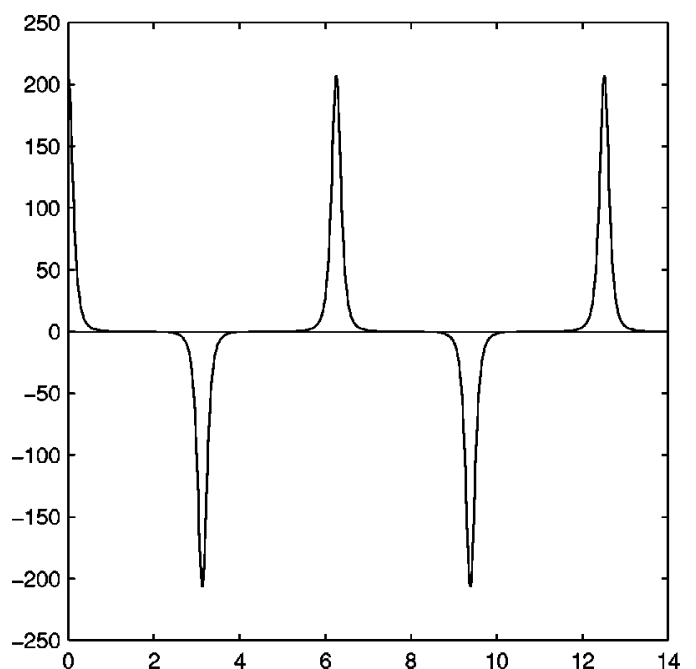

FIG. 4. Variation of vorticity across a cross section $(y=0)$ of the cell near the threshold value of $k, r=s=1, k=0.984$.

solution is that patterns of point vortices or finite regions of vorticity singularities are reached before the long wave limit is taken or attained.

For simplicity in algebra we focus on the case $r=s, k$ $=k_{1}$. Consider the point $(0,0)$. From Eq. (8), the hyperbolic tangent will attain the value one, and hence the value of the stream function will become infinite if

$$
\frac{1}{\left(1-k^{2}\right)^{1 / 4}}-\left(1-k^{2}\right)^{1 / 4}=2,
$$

or

$$
1-k^{2}=(\sqrt{2}-1)^{4}, \quad k \approx 0.985171 .
$$

Although the vorticity parameter $\sigma$ of Eq. (11) appears to be zero due to Eq. (13), the vorticity at $(0,0)$ is actually given by

$$
\begin{aligned}
\omega= & \frac{4 \sigma T\left(1+T^{2}\right)}{\left(1-T^{2}\right)^{2}}, \quad T=\tanh \frac{\psi}{4}, \quad T \rightarrow 1, \\
& \text { as } \quad k \rightarrow 0.985171,
\end{aligned}
$$

and hence the vorticity still possesses a simple pole at this particular value of $k$ given by Eq. (13).

It is instructive to study the actual transformation for the plots of vorticity as $k$ varies. Near $k=0.985171, \sigma$ is close to zero and the vorticity is very small except in the vicinity of $T=1$ (Fig. 4).

For still larger values of $k$, the stream function as given by (8) is not defined in a finite region within each cell, as the hyperbolic tangent must be less than one in absolute value. The boundary is defined by

$$
\begin{gathered}
{\left[\frac{\operatorname{dn}(r x, k)}{\left(1-k^{2}\right)^{1 / 4}}-\frac{\left(1-k^{2}\right)^{1 / 4}}{\operatorname{dn}(r x, k)}\right]\left[\frac{\operatorname{dn}(r y, k)}{\left(1-k^{2}\right)^{1 / 4}}-\frac{\left(1-k^{2}\right)^{1 / 4}}{\operatorname{dn}(r y, k)}\right]} \\
\quad=2\left(\frac{1}{\left(1-k^{2}\right)^{1 / 4}}-\left(1-k^{2}\right)^{1 / 4}\right) .
\end{gathered}
$$

The curve resembles a circle (Fig. 5).

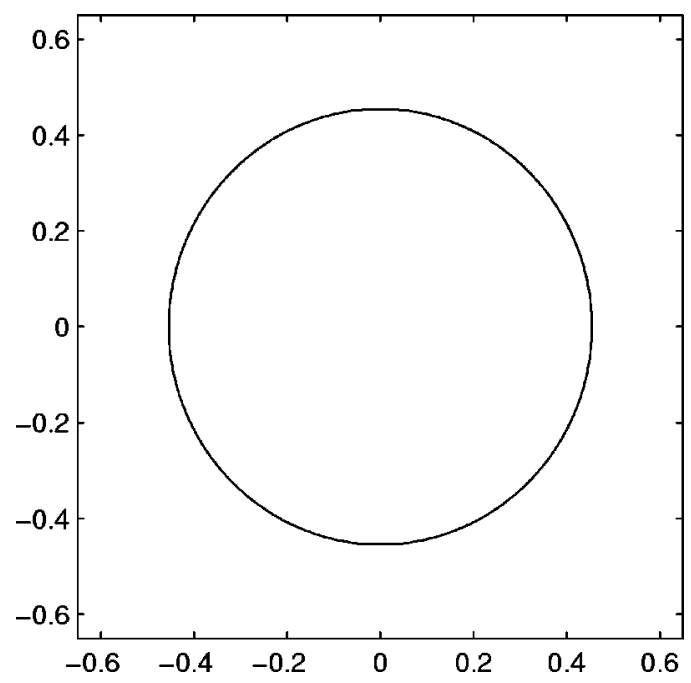

FIG. 5. Singularity boundary $(\tanh \psi / 4=1)$ beyond the threshold value of $k, r=s=1, k=0.99$.

The flow configuration beyond the critical value of $k$ defined by (14) requires careful consideration. Inside the critical contour the stream function given by (8) cannot be used since the hyperbolic tangent is larger than one in the interior. However, we propose that the flow field given by

$$
u=\psi_{y}, \quad v=-\psi_{x},
$$

is still a valid flow field, and the resulting vorticity remains finite. Mathematically the stream function would be complex constants in the interior streamlines. However, physical quantities, such as velocities (derivatives of the stream function) and mass flow (difference of adjacent streamlines) can still be calculated in a meaningful way. The vorticity field remains finite in the interior region. Hence one would obtain a "nearly circular singularity" in the vorticity field along the curve defined by Eq. (15) for $k$ beyond the critical value (of about 0.985171$)$.

One possible way to avoid a singularity is to consider a closed curve exterior to the boundary defined by (15). Outside the closed curve the sinh Poisson equation holds, while the interior is governed by a different principle of vorticity dynamics. The situation is then analogous to the case of the classical Lamb dipole. A brief account of this vortex pattern is given here for completeness. Basically the flow is irrotational outside a circle of radius $a$, but the vorticity is proportional to the stream function $(\psi)$ inside the circle. Matching $\psi$ and its derivative across the circle give the Lamb dipole $^{5,12-14}$

$$
\begin{aligned}
& \psi=-U\left(r-\frac{a^{2}}{r}\right) \sin \theta, \quad r>a, \\
& \psi=C J_{1}(k r) \sin \theta, \quad \omega=-C k^{2} J_{1}(k r) \sin \theta, \quad r<a, \\
& J_{1}(k a)=0, \quad C=\frac{-2 U}{k J_{0}(k a)} .
\end{aligned}
$$

$U$ is the free stream speed in the far field.

Special reduction and potential applications. The long wave limits of these doubly periodic patterns of vortex arrays 
will typically lead to known solutions. For simplicity we shall first take the solution equation (4) as an example. The cn function has period $4 K$, where $K$ is the complete elliptic integral of the first kind. The stream function is given by Eq. (4), and there are two equations relating the five parameters $r, s, k, k_{1}, \sigma$. The periods in the $x, y$ directions are $4 K / r$, $4 K_{1} / s$, respectively. $K_{1}$ is the complete elliptic integral with parameter $k_{1}$.

To construct a solution, one first selects (or is given) the parameters $k, k_{1}$. One then defines the period (or length of the box) and the vorticity by solving for $r, s$, and $\sigma$. Since only two equations are involved, one can further assign a value to one of $r, s$, and $\sigma$.

The long wave limit refers to the case of $k_{1}$ approaching one. An inspection of the equations shows that the finite, plausible solution is $k$ approaching zero, and this case reduces to the Mallier-Maslowe vortices.

The main formula of the present work [Eq. (8)] will also reduce to the Mallier-Maslowe vortices in the limits $k \rightarrow 0$ and $k_{1} \rightarrow 1$. These auxiliary results are needed

$$
\begin{aligned}
& \operatorname{dn}^{2}(r x)=1-k^{2} \operatorname{sn}^{2}(r x), \quad \operatorname{sn}(r x) \rightarrow \sin (r x) \text { as } k \rightarrow 0, \\
& \operatorname{dn}(s y) \rightarrow \operatorname{sech}(s y) \text { as } k_{1} \rightarrow 1 .
\end{aligned}
$$

On simplifying the algebra, (8) reduces to (for the case $\sigma$ $=1$ )

$$
\psi=4 \tanh ^{-1}\left[\frac{s \operatorname{sech}(s y) \cos (2 r x)}{2 r}\right], \quad 4 r^{2}=1+s^{2},
$$

which is a form of the Mallier-Maslowe vortices [Eq. (3)].

Numerical computations of two-dimensional, incompressible turbulence have revealed the emergence of coherent vortex structures. Such self-organization of the flow deserves attention, since vortex-wall interactons will eventually become important as the size of the vortices increases. ${ }^{15}$ Experiments on such quasi-two-dimensional flows in rotating ${ }^{16}$ or stratified ${ }^{17}$ fluids, or electromagnetically forced flows ${ }^{18}$ are often performed in containers of finite size and with rigid boundaries. Isolated or arrays of vortices resembling those found in the present work are observed, and hence the present formulation can enhance our capability in understanding such flows.

Arrays of vortices are indeed observed in these experimental situations. Especially striking are flows produced in a thin layer of electrolyte by using a steady, spatially periodic, electromagnetic forcing. ${ }^{19}$ The flow patterns resemble those predicted by the kind of analytical formulation in this paper.

Stability of the these vortices remains an open question. Methods similar to those used earlier in the literature ${ }^{3-5}$ can presumably be applied. Alternatively full scale numerical simulations should be performed. Indeed three-dimensional secondary instability of the Mallier-Maslowe vortices has been studied by computational methods, and related to features observed experimentally in shear flows and wakes. ${ }^{20}$

\section{ACKNOWLEDGMENT}

Partial financial support has been provided by the Research Grants Council through contracts HKU 7067/00E and HKU 7006/02E.

${ }^{1}$ D. Montgomery, W. H. Matthaeus, W. T. Stribling, D. Martinez, and S. Oughton, "Relaxation in two dimensions and the sinh-Poisson equation," Phys. Fluids A 4, 3 (1992).

${ }^{2}$ R. Mallier and S. A. Maslowe, "A row of counter rotating vortices," Phys. Fluids A 5, 1074 (1993).

${ }^{3}$ R. A. Pasmanter, "On long-lived vortices in 2D viscous flows, most probable states of inviscid 2D flows and soliton equation," Phys. Fluids 6, 1236 (1994).

${ }^{4}$ T. Dauxois, "Nonlinear stability of counter-rotating vortices," Phys. Fluids 6, 1625 (1994)

${ }^{5}$ T. Dauxois, S. Fauve, and L. Tuckerman, "Stability of periodic arrays of vortices," Phys. Fluids 8, 487 (1996).

${ }^{6} \mathrm{~K}$. W. Chow, N. W. M. Ko, and S. K. Tang, "Solitons in $(2+0)$ dimensions and their applications in vortex dynamics," Fluid Dyn. Res. 21, 101 (1997).

${ }^{7}$ K. W. Chow, N. W. M. Ko, R. C. K. Leung, and S. K. Tang, "Inviscid two dimensional vortex dynamics and a soliton expansion of the sinh-Poisson equation," Phys. Fluids 10, 1111 (1998).

${ }^{8}$ B. N. Kuvshinov and T. J. Schep, "Double-periodic arrays of vortices," Phys. Fluids 12, 3282 (2000).

${ }^{9} \mathrm{~K}$. W. Chow, "A class of doubly periodic waves for nonlinear evolution equations," Wave Motion 35, 71 (2002).

${ }^{10}$ M. J. Ablowitz and H. Segur, Solitons and the Inverse Scattering Transform (SIAM, Philadelphia, 1981).

${ }^{11}$ M. Abramowitz and I. Stegun, Handbook of Mathematical Functions (Dover, New York, 1964).

${ }^{12}$ A. H. Nielsen and J. Juul Rasmussen, "Formation and temporal evolution of the Lamb dipole," Phys. Fluids 9, 982 (1997).

${ }^{13}$ J. S. Hesthaven, J. P. Lynov, A. H. Nielsen, J. Juul Rasmussen, M. R. Schmidt, E. G. Shapiro, and S. K. Turitsyn, "Dynamics of a nonlinear dipole vortex," Phys. Fluids 7, 2220 (1995).

${ }^{14}$ V. V. Meleshko and G. J. F. van Heijst, "On Chaplygin's investigations of two dimensional vortex structures in an inviscid fluid," J. Fluid Mech. 272, 157 (1994).

${ }^{15}$ H. J. H. Clercx, S. R. Massen, and G. J. F. van Heijst, "Spontaneous spin-up during the decay of 2D turbulence in a square container with rigid boundaries," Phys. Rev. Lett. 80, 5129 (1998).

${ }^{16}$ E. J. Hopfinger and G. J. F. van Heijst, "Vortices in rotating fluids," Annu. Rev. Fluid Mech. 25, 241 (1993).

${ }^{17}$ B. M. Boubnov, S. B. Dalziel, and P. F. Linden, "Source-sink turbulence in a stratified fluid," J. Fluid Mech. 261, 273 (1994).

${ }^{18} \mathrm{P}$. Tabeling, S. Burkhart, O. Cardoso, and H. Willaime, "Experimental study of freely decaying two-dimensional turbulence," Phys. Rev. Lett. 67, 3772 (1991).

${ }^{19} \mathrm{O}$. Cardoso, D. Marteau, and P. Tabeling, "Quantitative experimental study of the free decay of quasi-two dimensional turbulence," Phys. Rev. E 49, 454 (1994).

${ }^{20}$ S. Julien, J. M. Chomaz, and J. C. Lasheras, "Three-dimensional stability of periodic arrays of counter-rotating vortices," Phys. Fluids 14, 732 (2002). 